\title{
Signalosomes in the brain: relevance in the development of certain neuropathologies such as Alzheimer's disease
}

\author{
Raquel Marin ${ }^{1,2,3 *}$ \\ 1 Laboratory of Cellular Neurobiology, Department of Physiology, School of Medicine, La Laguna University, Tenerife, Spain \\ 2 Institute of Biomedical Technologies, La Laguna University, Tenerife, Spain \\ ${ }^{3}$ Canarian Institute of Cancer Research, Tenerife, Spain
}

\section{Edited by:}

Ali Mobasheri, The University of

Nottingham, UK

\section{Reviewed by:}

Carmen Valenzuela, Instituto de Investigaciones Biomédicas CSIC-UAM, Spain

Elena Lnes Posse de Chaves,

University of Alberta, Canada

\section{*Correspondence:}

Raquel Marin, Laboratory of Cellular Neurobiology, Department of

Physiology, School of Medicine, La Laguna University, La Laguna 38320,

Tenerife, Spain.

e-mail:rmarin@ull.es
Emerging data suggest that compartmentalization of signaling molecules into particular membrane compartments, or lipid rafts, may be at the basis of numerous activities related to neuronal preservation against different pathologies. These signaling platforms (signalosomes) are formed by complex lipid and protein that may interact to develop a plethora of different physiological responses upon activation by different extracellular stimuli, thereby contributing to neuroprotection. One of the first studied signalosomes involved in neuroprotection against Alzheimer's disease (AD) is constituted by estrogen receptor (ER), in association with scaffolding caveolin-1 and a voltage-dependent anion channel (VDAC). In this complex, ER plays a neuroprotective role partially through the modulation of VDAC activation, a porin involved in amyloid-beta-induced toxicity. Interestingly, ER and VDAC interactions appear to be altered in lipid rafts of $A D$ brains, a phenomenon that may contribute to neuronal impairment. Alterations in lipid components of these subdomains may contribute to destabilization of this macrocomplex. These recent advances in the relevance of signaling platforms related to brain preservation, in particular against $A D$, are discussed in this work.

Keywords: signaling platforms in neurons, membrane estrogen receptor, voltage-dependent anion channel, neuroprotection Alzheimer's disease

\section{SIGNALING PLATFORMS IN NEURONAL LIPID RAFTS}

Neuronal signal transduction is initiated at the plasma membrane by a plethora of protein and lipid interactions which are crucial for the correct cellular communication and activities, and whose alterations may determine cognitive and neurological impairments. An accepted fact is that cognitive decline that occurs with normal aging, and is exacerbated in certain neuropathologies, is mainly related to functional changes in intracellular signaling cascades and cellular communication that alter normal neuronal responses. In these order of ideas, liquid-ordered microdomains of the plasma membrane, or lipid rafts, are a preferential location of many signaling proteins. These membrane subdomains are presently defined as dynamic assemblies of proteins and specific lipid classes, i.e., cholesterol, sphingolipids, gangliosides, in which different lipid-lipid, lipid-protein, and protein-protein oligomerizing interactions take place, thus stimulating raft stability (Simons and Gerl, 2010). In lipid rafts, molecules can move and interact with different kinetics in response to a variety of intra- and/or extracellular ligands that may ultimately favor specific protein interactions and signaling cascades activation. Therefore, the distribution of lipid hallmarks in these microstructures that modulate membrane fluidity and other physicochemical properties may affect protein dynamics, and presumably alter their function and signal transduction.

In this sense, many raft intrinsic proteins contain a lipid moiety contributing to their stabilization and functioning. For example, numerous proteins have a special post-translational modification known as the glycosylphosphatidylinositol (GPI) anchor, which serves to attach the proteins to the cell membrane and lipid rafts.
These GPI-protein interactions are crucial to initiate different signaling events within these subdomains (Paulick and Bertozzi, 2008). Other lipid modifications, such as palmitoylation, have been characterized in proteins which have an important role in neuronal maintenance, such as Src tyrosine kinases (SKTs; Sato et al., 2009) and steroid receptors (Pedram et al., 2007).

Apart from lipidic anchors, raft scaffolding proteins, such as caveolin family, may importantly contribute to the stable insertion of signaling proteins into neuronal lipid rafts (Parton, 2003). These proteins not only provide stabilizing scaffolds for lipid raft maintenance, but also serve to compartmentalize specific signaling molecules within these domains, with the prospect of rapidly and selectively modulating cell signaling events (Lajoie et al., 2009). Thus, caveolins have been shown to regulate a variety of key signaling elements, including G-proteins, and STK, phosphatidylinositol-3 kinase (PI3K) and mitogen-activated protein kinase (MAPK) pathways (Arcaro et al., 2007). Moreover, caveolins interact with the amyloid precursor protein (APP) modulating amyloidogenic processing (Ehehalt et al., 2003), and with membrane estrogen receptors (mERs; Boulware et al., 2007; Marin et al., 2008), thus modifying neuroprotective responses.

Lipid rafts are also platforms for neurotrophic signaling initiated by diverse neurotrophins which are essential for synaptic transmission, axon guidance, and cell adhesion (Tsui-Pierchala et al., 2002). In this sense, different neurotrophin and neurotransmitter receptors are located in these microstructures, and channel activity and properties appear to vary depending upon their location in rafts (Martens et al., 2000). 
Thus, lipid rafts within neuronal membranes can integrate protein signaling platforms or "signalosomes," which may be are crucial for the development of neuronal physiological activities.

\section{SIGNALOSOMES INVOLVED IN NEURONAL PRESERVATION: THE PARADIGM OF ER-VDAC-CAVEOLIN-1 COMPLEX RELATED TO AD}

It is widely accepted that estrogen develops some crucial actions in the regulation of brain development, plasticity, and preservation (Suzuki et al., 2006). Thus, estrogen signaling mechanisms have been demonstrated in neurogenesis, neuronal differentiation, synaptic plasticity, and neuroprotection, mainly in brain areas involved in mood and cognitive processes (Marin et al., 2005; Raz et al., 2008). These actions can be exerted through hormone binding to different cell surface targets, including mERs that activate rapid mechanisms of action (Vasudeban and Pfaff, 2008). Indeed, mER participates in neuroprotection against amyloid-beta $(A \beta)$-induced neurotoxicity (Marin et al., 2003), through the rapid estrogen activation of MAPK signaling pathway (Guerra et al., 2004). A $\beta$ aggregation is a main hallmark of Alzheimer's disease (AD) pathology, and is derived from the proteolysis of APP within lipid rafts (Vardy et al., 2005). Other data in different cell and animal models have corroborated the involvement of rapid mER actions in preventing $\mathrm{A} \beta$-induced degeneration (Pike et al., 2009).

A recent interesting finding is that $\mathrm{mER}$ is highly enriched in neuronal lipid rafts of different cognitive areas in human brains. This molecule, lacking large hydrophobic domains, is stabilized by interactions with caveolin-1 and other proteins, including plasmalemmal voltage-dependent anion channel (pl-VDAC) and the insulin growth factor-1 receptor (IGF-1R; Ramirez et al., 2009). These proteins integrated in a macromolecular complex may form a signaling platform involved in different neuroprotective activities against a variety of injuries (Marin et al., 2009). Indeed, present available evidences have demonstrated that estrogen receptor (ER) and IGF-1R pathways cross-talk with each other to promote neuroprotective events, and this association appears to be affected with aging (Garcia-Segura et al., 2007). Moreover, this macromolecular complex may be differentially activated by the natural ligands of these receptors, i.e., estrogens and IGF-1, thereby adapting the neuronal response to extracellular ligand availability that may modulate the intracellular signaling machinery (Marin et al., 2009). In support of this hypothesis, some data has evidenced that pl-VDAC activation and gating may be regulated by rapid actions of estrogens through phosphorylation processes (Diaz et al., 2001), and this may importantly contribute to neuronal survival against $\mathrm{AD}$ neurotoxicity. As observed in immunochemical studies, voltage-dependent anion channel (VDAC) is highly enriched in cortical lipid rafts of AD brains, and is abundant surrounding senile plaques in either AD mouse or human brains (Ferrer, 2009; Ramirez et al., 2009), suggesting a role for this porin in $\mathrm{AD}$ development. Furthermore, in cultured neurons, pl-VDAC has been shown to be involved in the mechanisms of $A \beta$-induced toxicity, observing a reduction of amyloid neurotoxicity following a physical closing of the channel (Marin et al., 2007). It has also been suggested that VDAC may interact with $A \beta$, thereby promoting its aggregation and contributing to neurotoxicity (Thinnes, 2010). Therefore, the regulation of pl-VDAC gating in this signalosome to maintain the channel inactivated may be crucial for neuronal preservation against $A \beta$ neurotoxicity. In this order of ideas, it has been demonstrated that estradiol triggers a PKA/Src kinase pathway to rapidly promote VDAC phosphorylation in neuronal membranes (Herrera et al., 2011a), a phenomenon that may increase estrogen cell survival against $\mathrm{A} \beta$ neurotoxicity (Herrera et al., 2011b). Altogether, these data suggest that ER/VDAC association in lipid rafts plays a pivotal role in preservation against $\mathrm{AD}$.

An emerging concept is that potential disruption of these signalosomes as a consequence of different factors affecting the homeostasis of lipid rafts may underpin the etiology of different neuropathologies. In this order of ideas, the molecular complex formed by ER, VDAC, and caveolin-1 appears to be disrupted in lipid rafts at late stages of $\mathrm{AD}$. Thus, in lipid rafts from cortical areas of AD brains, VDAC maintains, and even enhances, its association with caveolin-1 whereas ER is mainly enriched in astrocytes, in particular, surrounding amyloid plaques (Ramirez et al., 2009). The up-regulation of ER in astrocytes following an insult has been previously reported, suggesting a protective role of this receptor against inflammation (Lu et al., 2003). These protein rearrangements occurring in lipid rafts of AD brains may profoundly affect signaling responses for neuronal integrity, a possibility that requires further exploration.

A sustainable possibility is that aberrant interactions in multimolecular signaling complexes such as the one formed by ER, VDAC, IGF-1R, and caveolin-1 may be largely affected by alterations of lipid composition in lipid rafts, a fact that modifies the physicochemical properties of these microstructures. In the last years, the hypothesis that raft changes in lipid composition may contribute to neuropathologies has gained considerable support (Rushworth and Hooper, 2011). Thus, structural modifications in lipid rafts induced by alterations in cholesterol, ganglioside, and polyunsaturated fatty acids (PUFA) content result in the development of different aspects of AD pathology (Hashimoto et al., 2002; Martin et al., 2010). The biological relevance of raft-associated cholesterol and gangliosides related to different neurological diseases has been recently reviewed (Schengrund, 2010).

These changes may compromise lipid raft structure provoking lipid rearrangements which alter numerous properties of these structures, such as homeoviscous state, unsaturation index, peroxidability, and fluidity (Aksenov et al., 2001). Alterations in the physicochemical properties of lipid rafts may also affect structure-activity relationships of proteins involved in a broad range of neurodegenerative diseases, including, apart from $\mathrm{AD}$, prion diseases, Huntington's disease, amyotrophic lateral sclerosis, and Parkinson's disease (Taylor and Hooper, 2007; Schengrund, 2010).

Additional support for this concept is starting to be provided by the observed integration in lipid rafts of proteins which are hallmarks of different pathogenesis, such as the prion protein $\left(\operatorname{PrP}^{\mathrm{c}}\right)$, alpha synuclein and parkin (Park et al., 2009; Pani et al., 2010; Schengrund, 2010). Thus, $\operatorname{PrP}^{c}$ is constitutively expressed in neurons as a GPI-anchored protein localized in lipid rafts, and these compartments play an important role in the propagation of prion disease (Russelakis-Caneiro et al., 2004; Morris et al., 2006). Moreover, alpha synuclein also interacts with these membrane compartments, and raft disruption relocates this protein away from 
synapses probably compromising neurotransmission (Fortin et al., 2004). These preliminary studies are worthy of pursuing to further explore the importance of lipid rafts in the activities of these protein markers that contribute to neurotoxicity.

Overall, these findings reflect the importance of lipid raft platforms in normal neuronal functionality and preservation, and the dramatic consequences of raft deregulation as a result of impairment in the physicochemical properties of these microenvironments. Undoubtedly, further work on these interactive microstructures will unveil important aspects of numerous neurological diseases.

\section{PERSPECTIVES}

An increasing number of reports indicate that compartmentalization of signaling macrocomplexes in lipid rafts have a pivotal role in the regulation of distinct markers directed to neuronal preservation. These signalosomes may become activated upon association with a variety of stimuli, leading to the initiation of different signal transduction events that may promote neuronal survival against different injuries. Although some of the components of these platforms are starting to be identified, the complete picture is surely more complex, involving alterations in different signaling markers whose adverse and/or beneficial consequences remain to be clarified. An example of this is the identified interaction of ER, known to develop neuroprotective actions, and VDAC, recently identified as a contributor to $A \beta$ neurotoxicity, which are assembled in a multimolecular signaling complex in neuronal lipid rafts. No doubt that other still unidentified components of this signaling complex participating in neuroprotection remain to be characterized. Furthermore, an important aspect is that altered composition of different lipid classes integrated in these membrane compartments contributes to impaired signaling mechanisms, and may underlie some of the neuropathological parameters. Therefore, identification of crucial lipid markers and their interactions with target signaling proteins in lipid rafts are an important challenge to elucidating the mechanisms involved in neuronal pathophysiologies. Understanding these complex, diverse, and interactive molecular assemblies will undoubtedly provide the clues to the development of future therapies against these neurological diseases.

\section{ACKNOWLEDGMENT}

Supported by grants SAF2010-22114-C02-01/02.

\section{REFERENCES}

Aksenov, M. Y., Aksenova, M. V., Butterfield, D. A., Geddes, J. W., and Markesbery, W. R. (2001). Protein oxidation in the brain in Alzheimer's disease. Neuroscience 103, 373-383.

Arcaro, A., Aubert, M., Espinosa del Hierro, M. E., Khanzada, U. K., Angelidou, S., Tetely, T. D., Bittermann, A. G., Frame, M. C., and Seckl, M. J. (2007). Critical role for lipid raftassociated Src kinases in activation of PI3K-Akt signaling. Cell. Signal. 19, 1081-1092.

Boulware, M. I., Kordasiewicz J., and Mermelstein, P. G. (2007). Caveolin proteins are essential for distinct effects of membrane estrogen receptors in neurons. J. Neurosci. 27, 9941-9950.

Diaz, M., Bahamonde, M. I., Lock, H., Muñoz, F. J., Hardy, S. P., Posas, F., and Valverde, M. A. (2001). Okadaic acid-sensitive activation of Maxi $\mathrm{Cl}(-)$ channels by triphenylethylene antioestrogens in C1300 mouse neuroblastoma cells. J. Physiol. 536, 79-88.

Ehehalt, R., Keller, P., Haass, C., Thiele, C., and Simons, K. (2003). Amyloidogenic processing of the Alzheimer betaamyloid precursor protein depends on lipid rafts. J. Cell Biol. 160, 113-123.

Ferrer, I. (2009). Altered mitochondria, energy metabolism, voltagedependent anion channel, and lipid rafts converge to exhaust neurons in Alzheimer's disease. J. Bioenerg. Biomembr. 41, 425-431.

Fortin, D. L., Troyer, M. D., Nakamura, K., Kubo, S.,Anthony, M.D., and Edwards, R. H. (2004). Lipid rafts mediate the synaptic localization of $\alpha$-synuclein. Neurobiol. Dis. 24, 6715-6723.

Garcia-Segura, L. M., Diz-Chaves, Y., Perez-Martin, M., and Darnaudery, M. (2007). Estradiol, insulin-like growth factor-1 and brain aging. Psychoneuroendocrinology 32, 557-561.

Guerra, B., Diaz, M., Alonso, R., and Marin, R. (2004). Plasma membrane oestrogen receptor mediates neuroprotection against $\beta$-amyloid toxicity through activation of Raf1/MEK/ERK cascade in septal-derived cholinergic SN56 cells. J. Neurochem. 91, 99-109.

Hashimoto, M., Hossain, S., Shimada, T., Sugioka, K., Yamasaki, H., Fujii, Y., Ishibashi, Y., Oka, J. I., and Shido, O. (2002). Docosahexaenoic acid provides protection from impairment of learning ability in Alzheimer's disease model rats. J. Neurochem. 81, 1084-1091.

Herrera, J. L., Díaz, M., HernándezFernaud, J. R., Salido, E., Alonso, R., Fernández, C., Morales, A., and Marin, R. (2011a). Voltage-dependent anion channel as a resident protein of lipid rafts: pots-transductional regulation by estrogens and involvement in neuronal preservation against Alzheimer's disease. J. Neurochem. 116, 820-827.

Herrera, J. L., Fernandez, C. E., Diaz, M., Cury, D., and Marin, R. (2011b). Estradiol and tamoxifen differentially regulate a voltage-dependent anion channel involved in amyloid-beta induced neurotoxicity. Steroids. doi: 10.1016/j.steroids.2011.02.014

Lajoie P., Goetz, J. G., Dennis, J. W., and Nabi, I. R. (2009). Lattices, rafts, and scaffolds: domain regulation of receptor signaling at the plasma membrane. J. Cell Biol. 185, 381-385.

Lu, Y.-P., Zeng, M., Hu, X.-Y., Xu, H., Swaab, D. F., Ravid, R., and Zhou, J.-N. (2003). Estrogen receptor $\alpha$-immunoreactive astrocytes are increased in the hippocampus in Alzheimer's disease. Exp. Neurol. 183, 482-488.

Marin, R., Diaz, M., Alonso, R., Sanz, A., Arevalo, M. A., and Garcia-Segura, L. M. (2009). Role of estrogen receptor alpha in membrane-initiated signaling in neural cells: interaction with IGF-1 receptor. J. Steroid Biochem. Mol. Biol. $114,2-7$.

Marin, R., Guerra, B., Alonso, R., Ramírez, C. M., and Díaz M. (2005). Estrogen activates classical and alternative mechanisms to orchestrate neuroprotection. Curr. Neurovasc. Res. 2, 287-301.

Marin, R., Guerra, B., Morales, A., Diaz, M., and Alonso, R. (2003). An oestrogen membrane receptor participates in estradiol actions for the prevention of amyloid- $\beta$ peptide 1-40-induced toxicity in septal-derived cholinergic SN56 cells. J. Neurochem. 85, 1180-1189.

Marin, R., Ramírez, C. M., González, M., González-Muñoz, E., Zorzano, A., Camps, M., Alonso R., and Díaz M. (2007). Voltage-dependent anion channel (VDAC) participates in amyloid beta-induced toxicity and interacts with plasma membrane estrogen receptor $\alpha$ in septal and hippocampal neurons. Mol. Membr. Biol. 24, 148-160.
Marin, R., Ramirez, C. M., Morales, A., Gonzalez, M., Alonso, R., and Diaz, M. (2008). Modulation of $\mathrm{A} \beta$-induced neurotoxicity by estrogen receptor alpha and other associated proteins in lipid rafts. Steroids 73, 992-996.

Martens, J. R., Navarro-Polanco, R., Coppock, E. A., Nishiyama, A., Parshley, L., Grobaski, T. D., and Tamkun, M. M. (2000). Differential targeting of shakerlike potassium channels to lipid rafts. J. Biol. Chem. 275, 7443-7446.

Martin, V., Fabelo, N., Santpere, G., Puig, B., Marin, R., Ferrer, I., and Diaz, M. (2010). Lipid alterations in lipid rafts from Alzheimer's disease human brain cortex. J. Alzheimers Dis. 19, 489-502.

Morris, R. J., Parkyn, C. J., and Jen, A. (2006). Traffic of prion protein between different compartments on the neuronal surface, and the propagation of prion disease. FEBS Lett. 580, 5565-5571.

Pani, A., Mandas, A., and Dessì, S. (2010). Cholesterol,Alzheimer's disease, prion disorders: a ménage à trois? Curr. Drug Targets 11, 1018-1031.

Park, J. Y., Kim, K. S., Lee, S. B., Ryu, J. S., Chung, K. C., Choo, Y. K., Jou, I., Kim, J., and Park, S. M. (2009). On the mechanism of internalization of alpha-synuclein into microglia: roles of ganglioside GM1 and lipid raft. J. Neurochem. 110, 400-411.

Parton, R. G. (2003). Caveolae-form ultrastructure to molecular mechanisms. Nat. Rev. Mol. Cell Biol. 4, 162-167. Paulick, M. G., and Bertozzi, C. R. (2008). The glycosylphos- 
phatidylinositol anchor: a complex membrane-anchoring structure for proteins. Biochemistry 47, 6991-7000.

Pedram, A., Razandi, M., Sainson, R. C., Kim, J. K., Hughes, C. C., and Levin, E. R. (2007). A conserved mechanism for steroid receptor translocation to the plasma membrane. J. Biol. Chem. 282, 22278-22288.

Pike, C. J., Carroll, J. C., Rosario, E. R., and Barron, A. M. (2009). Protective actions of sex steroid hormones in Alzheimer's disease. Front. Neuroendocrinol. 3, 239-258.

Ramirez, C. M., Gonzalez, M., Diaz, M., Alonso, R., Ferrer, I., Santpere, G., Puig, B., Meyer G., and Marin, R. (2009). VDAC and ER $\alpha$ interaction in caveolae from human cortex is altered in Alzheimer's disease. Mol. Cell. Neurosci. 42, 172-183.

Raz, L., Khan, M. M., Mahesh, V. B., Vadlamudi, R. K., and Brann, D. W. (2008). Rapid estrogen signalling in the brain. Neurosignals 16, 140-153.
Rushworth, J. V., and Hooper, N. M. (2011). Lipid rafts: linking Alzheimer's amyloid- $\beta$ production, aggregation, and toxicity at neuronal membranes. Int. J.Alzheimers Dis. doi: $10.4061 / 2011 / 603052$

Russelakis-Caneiro, M., Hetz, C., Maundrell, K., and Soto C. (2004). Prion replication alters the distribution of synaptophysin and caveolin 1 in neuronal lipid rafts. Am. J. Pathol. 165, 1839-1848.

Sato I., Obata Y., Kasahara K., Nakayama, Y., Fukumoto, Y., Yamasaki, T., Yokohama, K. K., Saito, T., and Yamaguchi, N. (2009). Differential trafficking of Src, Lyn, Yes and Fyn is specified by the state of palmitoylation in the SH4 domain. J. Cell Sci. 122, 965-975.

Schengrund, C. L. (2010). Lipid rafts: keys to neurodegeneration. Brain Res. Bull. $82,7-17$.

Simons, K., and Gerl, M. J. (2010). Revitalizing membrane rafts: new tools and insights. Nat. Rev. 11, 688-699.
Suzuki, S., Brown, C. M., and Wise, P. M. (2006). Mechanisms of neuroprotection by estrogen. Endocrine 29, 209-215.

Taylor, D. R., and Hooper, N. M. (2007). Role of lipid rafts in the processing of the pathogenic prion and Alzheimer's amyloid-beta proteins. Semin. Cell Dev. Biol. 18, 638-648.

Thinnes, F. P. (2010). A $\beta$, cut from APP by $\beta$-secretase BACE 1 and $\gamma$-secretase, induces apoptosis via opening type-1 porin/VDAC in cell membranes of hypometabolic cells - a basic model for the induction of apoptosis? $\mathrm{Mol}$. Genet. Metab. 101, 301-303.

Tsui-Pierchala, B., Encinas, M., Milbrandt, J., and Jonson, E. M. (2002). Lipid rafts in neuronal signaling and function. Trends Neurosci. 25, 412-417.

Vardy, E. R. L. C., Catto, A. J., and Hooper, N.M. (2005). Proteolytic mechanisms in amyloid- $\beta$ metabolism: therapeutic implications for Alzheimer's disease. Trends Mol. Med. 11, 464-472.

Vasudeban, N., and Pfaff, D. W. (2008) Non-genomic actions of estrogens and their interaction with genomic actions in the brain. Front. Neuroendocrinol. 29, 238-257.

Conflict of Interest Statement: The author declares that the research was conducted in the absence of any commercial or financial relationships that could be construed as a potential conflict of interest.

Received: 21 March 2011; paper pending published: 11 April 2011; accepted: 10 May 2011; published online: 03 June 2011.

Citation: Marin R (2011) Signalosomes in the brain: relevance in the development of certain neuropathologies such as Alzheimer's disease. Front. Physio. 2:23. doi: 10.3389/fphys.2011.00023

This article was submitted to Frontiers in Membrane Physiology and Biophysics, a specialty of Frontiers in Physiology.

Copyright (c) 2011 Marin. This is an openaccess article subject to a non-exclusive license between the authors and Frontiers Media $S A$, which permits use, distribution and reproduction in other forums, provided the original authors and source are credited and other Frontiers conditions are complied with 